\title{
DESPRENDIMIENTO COROIDEO ASOCIADO A HIPOTONÍA OCULAR ESPONTÁNEA TARDÍA
}

\section{CHOROIDAL DETACHMENT ASSOCIATED WITH DELAYED SPONTANEOUS OCULAR HYPOTONY}

\author{
BOJADÓS $\mathrm{S}^{1}$, VELA JI ${ }^{1}$, ROSELLÓ $\mathrm{N}^{1}$, DÍAZ J ${ }^{1}$, BUIL JA ${ }^{2}$
}

\section{RESUMEN}

Casos clínicos: Se describen dos casos de hipotonía espontánea tardía asociada a desprendimiento coroideo (DC) y maculopatía hipotónica. Ambos pacientes habían sido operados de catarata 10 y 6 años antes del episodio de hipotonía respectivamente. En uno de ellos se combinó trabeculectomía. Ambos estaban recibiendo tratamiento con timolol en el momento de la presentación.

Discusión: La hipotonía espontánea tardía, sin cirugía ocular o un traumatismo reciente, representa un difícil diagnóstico. Se analizan las posibles causas.

Palabras clave: Hipotonía, desprendimiento coroideo, maculopatía hipotónica, timolol, cirugía filtrante.

\section{INTRODUCCIÓN}

La hipotonía ocular se define por la presencia de una presión intraocular (PIO) menor de $6 \mathrm{mmHg}$ o por la existencia de una PIO por debajo de un nivel que supone cambios funcionales y estructurales que impiden el normal funcionamiento del ojo (1).

\section{ABSTRACT}

Case reports: We report two cases of delayed spontaneous hypotony associated with choroidal detachment and hypotony maculopathy. Both patients had undergone uneventful cataract extractions 10 and 6 years prior to the occurrence of apparently spontaneous hypotony. Trabeculectomy was combined with phacoemulsification in one of them. Both had been receiving timolol at the time of presentation.

Discussion: Delayed spontaneous hypotony, without recent ocular surgery or trauma, represented a challenge in both the diagnosis and management processes in these patients. We have analysed the main causes (Arch Soc Esp Oftalmol 2007; 82: 381 384).

Key words: Hypotony, choroidal detachment, hypotony maculopathy, timolol, filtration surgery.

Recibido: 31/5/06. Aceptado: 24/52/07.

Servicio de Oftalmología. Hospital de la Santa Creu i de Sant Pau. Universidad de Barcelona. España.

1 Licenciado en Medicina.

2 Doctor en Medicina.

Correspondencia:

Sabrina Bojadós Puertas

Rambla Marina, $4264 .^{\circ} 1 .^{\mathrm{a}}$

08907 L'Hospitalet de Llobregat (Barcelona)

España

E-mail: sabrinabojados@ hotmail.com

Ocurre en diversas circunstancias clínicas, y puede deberse a causas reversibles o irreversibles. Suelen ser procesos oculares unilaterales, la mayoría durante el postoperatorio inmediato tras una cirugía de glaucoma. Se presentan dos casos de hipotonía espontánea tardía asociada a desprendimiento coroideo y maculopatía hipotónica. 
Ambos pacientes recibían tratamiento hipotensor con timolol. En ambos pacientes la causa de la hipotonía se atribuye a la sensibilidad al betabloqueante.

\section{CASOS CLÍNICOS}

\section{Caso clínico 1}

Mujer de 40 años que acudió a urgencias por disminución de agudeza visual (AV) de ojo derecho (OD) de una semana de evolución. Entre los antecedentes patológicos destacaba retraso psicomotor. Presentaba miopía magna, pseudofaquia bilateral (1995) y dos intervenciones de desprendimiento de retina en ojo izquierdo (OI) (2001). En la última visita realizada 6 meses antes presentaba una AV de contar dedos, PIO de $14 \mathrm{mmHg}$ y retina aplicada en dicho ojo. Su tratamiento actual era timolol en OD. La AV OD era de percepción de luz (PL) y en OI de noPL, la PIO era de $4 \mathrm{mmHg}$ en OD y $20 \mathrm{mmHg}$ en OI. La biomicroscopía y la gonioscopía eran normales. Se realizó un fondo de ojo (FO) (figs. 1 y 2) y una ecografía modo B (fig. 3) que evidenciaron un DC $360^{\circ}$ periférico. Se retiró el timolol y se pautó Pred-forte ${ }^{\circledR}$ cada dos horas y Dacortin ${ }^{\circledR} 45 \mathrm{mg}$ al día. Se observó una resolución total del DC (fig. 4) y un aumento de la PIO a $34 \mathrm{mmHg}$, por lo que se administró Azopt ${ }^{\circledR}$. Actualmente, 5 meses después, presenta una PIO de $22 \mathrm{mmHg}$ y una AV de movimiento de manos.

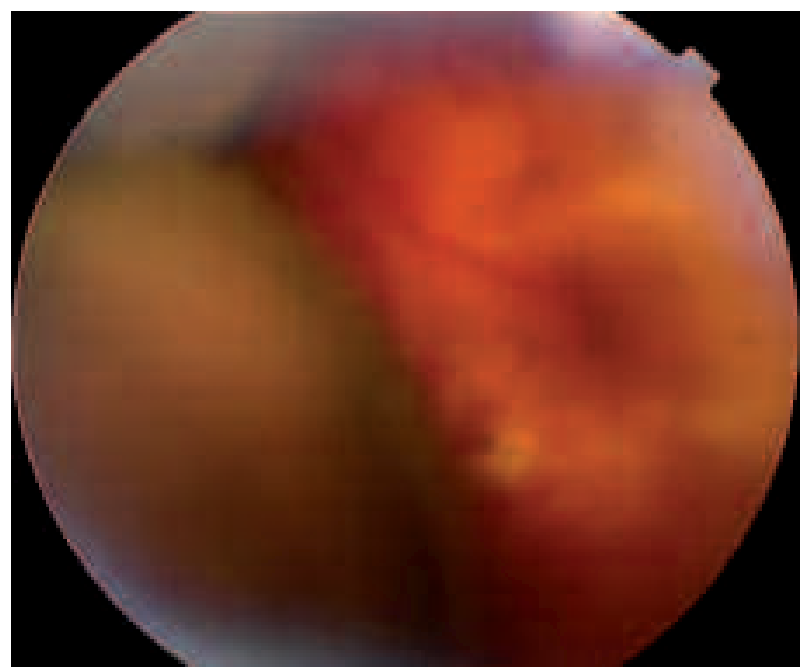

\section{Caso clínico 2}

Mujer de 68 años que presentaba afaquia bilateral e intervención de trabeculectomía con mitomicina en ambos ojos (AO) hacía 6 años. En los controles sucesivos semestrales se objetivó estabilidad visual y de la PIO en AO. Los últimos controles evidenciaron presiones de 22-24 mmHg por lo que se decidió instaurar tratamiento con timolol en AO. 2 meses después la paciente acudió presentando una AV de 0,3 en OD y 0,1 en OI, la PIO $10 \mathrm{mmHg}$ en OD y $2 \mathrm{mmHg}$ en OI. La biomicroscopía y la gonioscopía eran normales. En el FO se apreciaba un DC y pliegues maculares en OI (fig. 5). Mediante tomografía

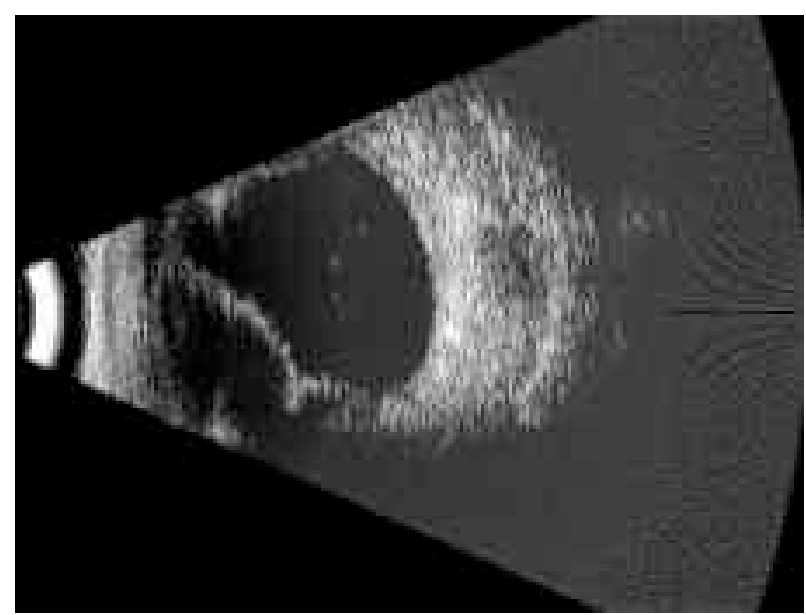

Fig. 3: Ecografía modo B del mismo paciente que demuestra el DC seroso.

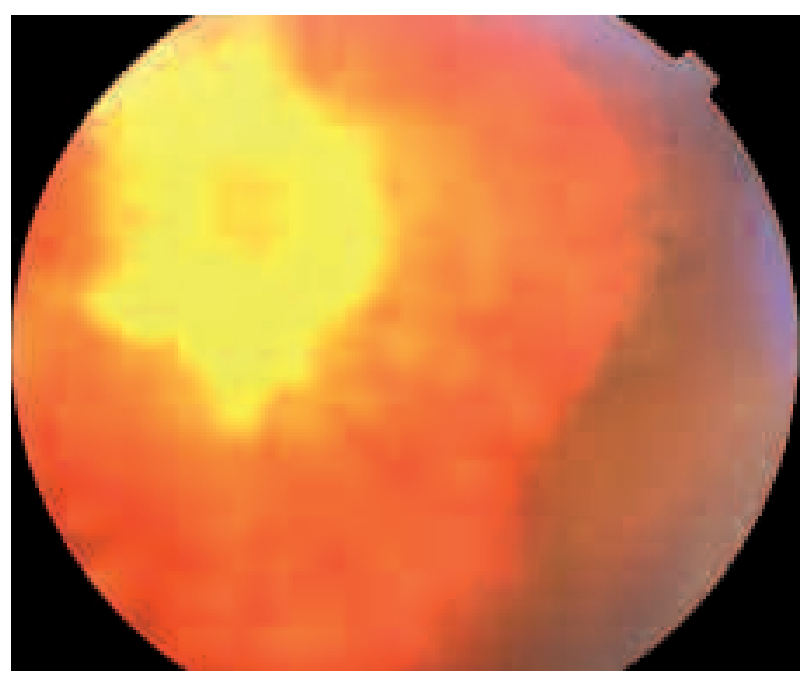

Fig. 1 y 2: Caso clínico 1: retinopatía miópica con desprendimiento coroideo $360^{\circ}$ periférico. 
de coherencia óptica (OCT) se objetivaron pliegues coriorretinianos (fig. 6), confirmándose la presencia de maculopatía hipotónica. Se retiró el timolol y se añadió Pred-forte ${ }^{\circledR}$ cada 2 horas. A los dos meses la PIO era de $11 \mathrm{mmHg}$ y la AV de 0,1. Actualmente se encuentra sin tratamiento.

\section{DISCUSIÓN}

La hipotonía es un hallazgo relativamente frecuente en la consulta del oftalmólogo. La causa más

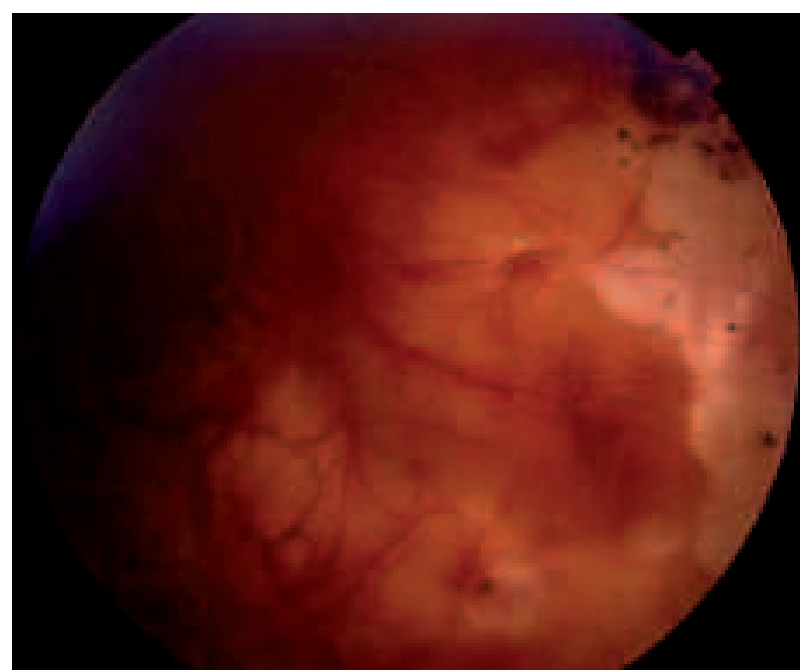

Fig. 4: Se aprecia la resolución del DC periférico. frecuente es una cirugía filtrante reciente (normalmente tras trabeculectomía con el uso de mitomicina). La hipotonía tardía, sin cirugía reciente o un traumatismo documentado, representa un reto diagnóstico.

Parece que la cirugía ocular predispone a la aparición de DC en combinación con hipotensores, de hecho hay descritos casos raros de DC en pacientes intervenidos de cataratas y tratados con dorzolamida o análogos de prostaglandinas (2).

El DC es una consecuencia de la hipotonía pero, una vez se ha instaurado, la perpetúa. El aumento de la PIO rompe dicho círculo vicioso (3). Su tratamiento se basa en corregir la causa subyacente. En primer lugar se debe retirar el tratamiento hipotensor. Los corticoides pueden elevar la PIO y disminuyen la inflamación del cuerpo ciliar, aunque su

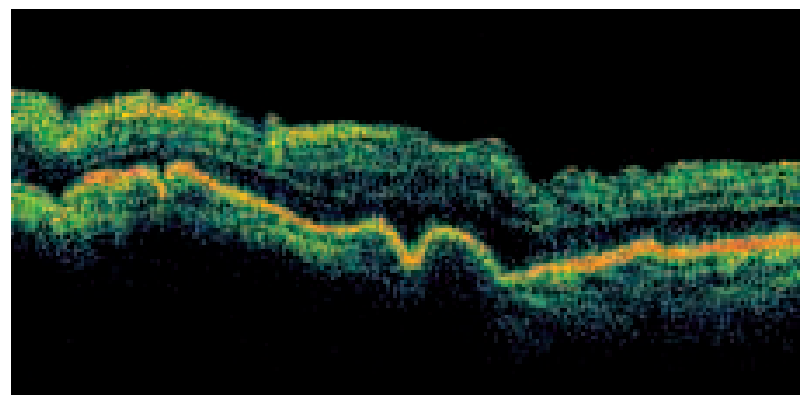

Fig. 6: OCT: pliegues coriorretinianos del mismo paciente. Corte efectuado a nivel macular.

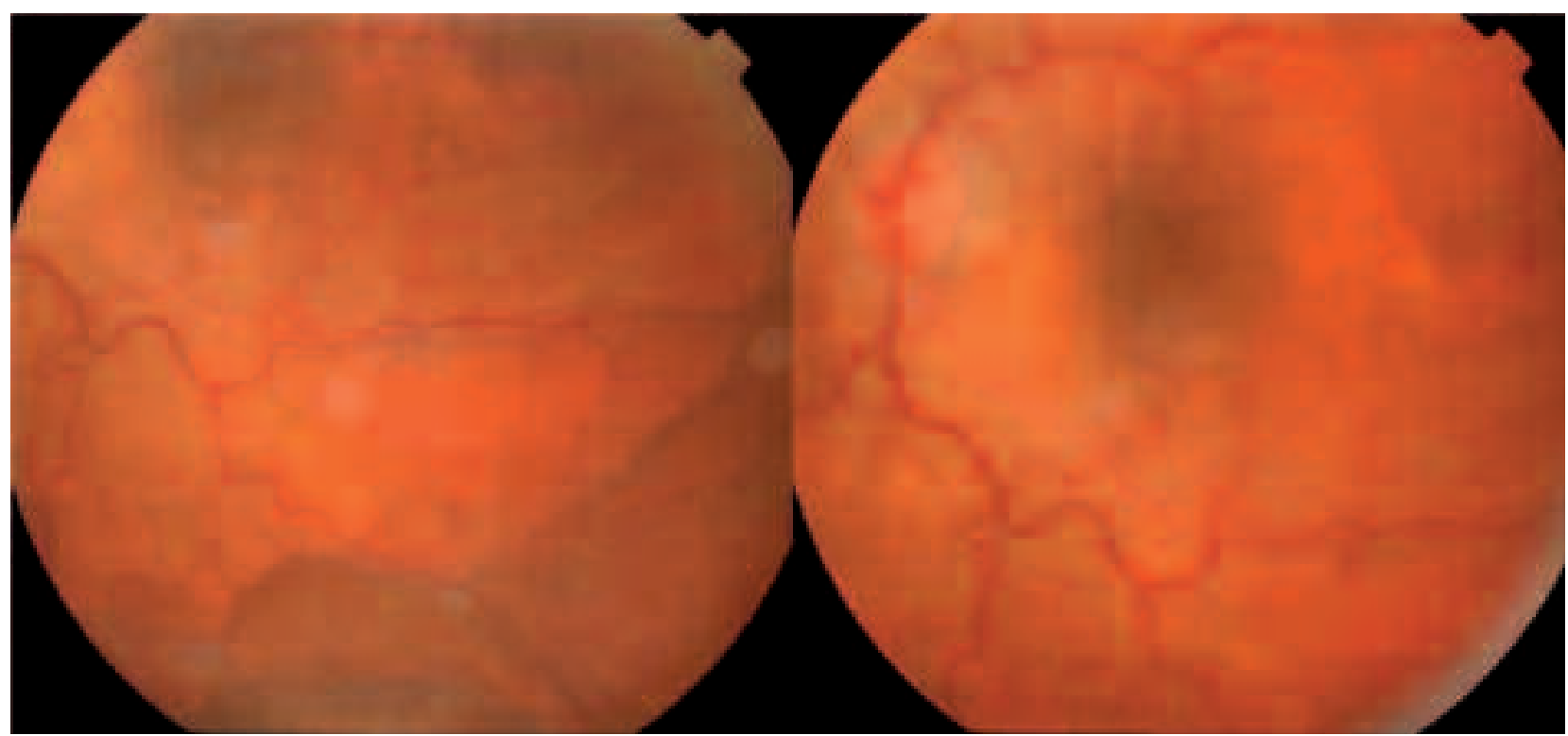

Fig. 5: Caso clínico 2. Se observan pliegues maculares debidos a la hipotonía y el DC en OI. 
indicación es controvertida en la hipotonía por ciclodiálisis; en cambio, la cicloplejia sí juega un papel importante en estos casos (4). Últimamente hay buenos resultados tras la inyección de BSS, viscoelásticos o gas (3). En última opción quedaría el tratamiento con láser (se ha utilizado láser diodo transescleral en casos de ciclodiálisis o sobre el trabécula para provocar su esclerosis) o quirúrgico (disección de proliferaciones epiciliares mediante vitrectomía, sutura quirúrgica de ciclodiálisis...).

En ambos pacientes la causa de la hipotonía tardía se atribuye a su tratamiento hipotensor. Existen pocos casos en la literatura de hipotonía tras tratamiento con betabloqueantes, inhibidores de la anhidrasa carbónica y análogos de prostaglandinas después de cirugías filtrantes o implantación de dispositivos de drenaje. Su etiopatogenia es controvertida. A parte de su acción reductora de la PIO, se ha propuesto la sensibilización del cuerpo ciliar tras una terapia crónica previa a la cirugía que provocaría una hipotonía al reinstaurar dicho tratamiento hipotensor (5).

La maculopatía hipotónica es una de las causas de disminución de AV por hipotonía.
En conclusión, la presencia de un desprendimiento coroideo asociado a una hipotonía sin cirugía o traumatismo recientes representa un desafío diagnóstico y terapéutico. Ante una hipotonía espontánea debemos identificar y corregir precozmente la causa para evitar la prolongación de sus efectos y complicaciones.

\section{BIBLIOGRAFÍA}

1. Budenz DL, Schartz K, Gedde SJ. Occult hypotony maculopathy diagnosed with optical coherence tomography. Arch Ophthalmol 2005; 123: 113-114.

2. Davani S, Delbosc B, Royer B, Kantelip JP. Choroidal detachment induced by dorzolamide 20 years after cataract surgery. Br J Ophtalmol 2002; 86: 1457-1458.

3. Mantel I, Schipper I. A simple treatment for chronic postoperative hypotony of unknown etiology: the injection of balances salt solution into the anterior chamber. Ophtalmic Surg Laser 2001; 32: 250-254.

4. Aminlari A, Callahan CE. Medical, laser, and surgical management of inadvertent cyclodyalisis cleft with hypotony. Arch Ophthalmol 2004; 122: 399-404.

5. Callahan C, Ayyala RS. Hypotony and choroidal effusion induced by topical timolol and dorzolamide in patients with previous glaucoma drainage device implantation. Ophthalmic Surg Lasers Imaging 2003; 34: 467-469. 